\title{
Endoscopically Applied Biodegradable Stent in a Rabbit Model of Pediatric Tracheomalacia
}

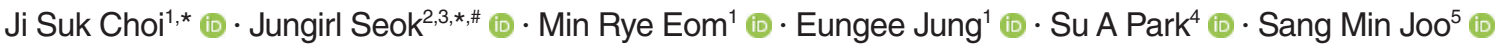 \\ Yeo Jin Jun ${ }^{5}$ (D) $\cdot$ Kil Won Son ${ }^{5}$ (D) $\cdot$ Seong Keun Kwon ${ }^{1,2,3}$ (i) \\ ${ }^{l}$ Department of Otorhinolaryngology-Head and Neck Surgery, Biomedical Research Institute, Seoul National University Hospital, Seoul; \\ ${ }^{2}$ Department of Otorhinolaryngology-Head and Neck Surgery, Seoul National University College of Medicine, Seoul; ${ }^{3}$ Department of Biomedical \\ Engineering, Seoul National University College of Medicine, Seoul; ${ }^{4}$ Department of Nature-Inspired Nanoconvergence Systems, Korea Institute of \\ Machinery and Materials, Daejeon; ${ }^{5}$ Taewoong Medical Co., Ltd., Gimpo, Korea
}

Objectives. A polydioxanone (PDO) stent was developed to treat tracheomalacia in pediatric patients. However, its safety and efficacy need to be verified in animal studies before clinical trials in patients can be conducted. This study evaluated the safety and efficacy of a PDO stent in normal and tracheomalacia-model rabbits.

Methods. In total, 29 New Zealand white rabbits were used: 13 for evaluating the biocompatibility of the PDO stent in normal rabbits and 16 for the creation of a tracheomalacia model. The tracheomalacia model was successfully established in 12 rabbits, and PDO stents were placed in eight of those rabbits.

Results. The PDO stent was successfully positioned in the trachea of the normal rabbits using an endoscopic approach, and its degradation was observed 10 weeks later. The stent fragments did not induce distal airway obstruction or damage, and the mucosal changes that occurred after stent placement were reversed after degradation. The same procedure was performed on the tracheomalacia-model rabbits. The survival duration of the tracheomalacia rabbits with and without stents was $49.0 \pm 6.8$ and $1.0 \pm 0.8$ days, respectively. Thus, the PDO stent yielded a significant survival gain $(P=0.001)$. In the tracheomalacia rabbits, stent degradation and granulation tissue were observed 7 weeks after placement, leading to airway collapse and death.

Conclusion. We successfully developed a PDO stent and an endoscopic guide placement system. The degradation time of the stent was around 10 weeks in normal rabbits, and its degradation was accelerated in the tracheomalacia model. The mucosal changes associated with PDO stent placement were reversible. Placement of the PDO stent prolonged survival in tracheomalacia-model rabbits.

Keywords. Tracheomalacia; Therapeutics; Trachea; Stents; Absorbable Implants; Polydioxanone; Pediatrics

\section{INTRODUCTION}

- Received July 30, 2020

Revised September 20, 2020

Accepted September 26, 2020

- Corresponding author: Seong Keun Kwon

Department of Otorhinolaryngology-Head and Neck Surgery, Seoul

National University Hospital, 101 Daehak-ro, Jongno-gu, Seoul 03080, Korea

Tel: +82-2-2072-2286, Fax: +82-2-745-2387

E-mail: otolarynx@snuh.org

*These authors contributed equally to this work.

${ }^{\#}$ Current affiliation: Department of Otorhinolaryngology-Head and Neck Surgery, National Cancer Center, Goyang, Korea
Tracheomalacia is characterized by weakness of the trachea due to reduction and/or atrophy of the longitudinal elastic fibers of the pars membrane or impaired cartilage integrity [1]. It is a rare condition, with uncertain incidence and prevalence. A singlecenter study revealed an estimated prevalence of one in 2,100 children [2]. The treatment of tracheomalacia depends on the severity of symptoms. Mild primary tracheomalacia in pediatric patients is preferentially treated by nonsurgical means, but aggressive therapy is required in severe tracheomalacia for which conservative measures are inadequate [3].

Copyright (C) 2021 by Korean Society of Otorhinolaryngology-Head and Neck Surgery.

This is an open-access article distributed under the terms of the Creative Commons Attribution Non-Commercial License (https://creativecommons.org/licenses/by-nc/4.0)

which permits unrestricted non-commercial use, distribution, and reproduction in any medium, provided the original work is properly cited. 

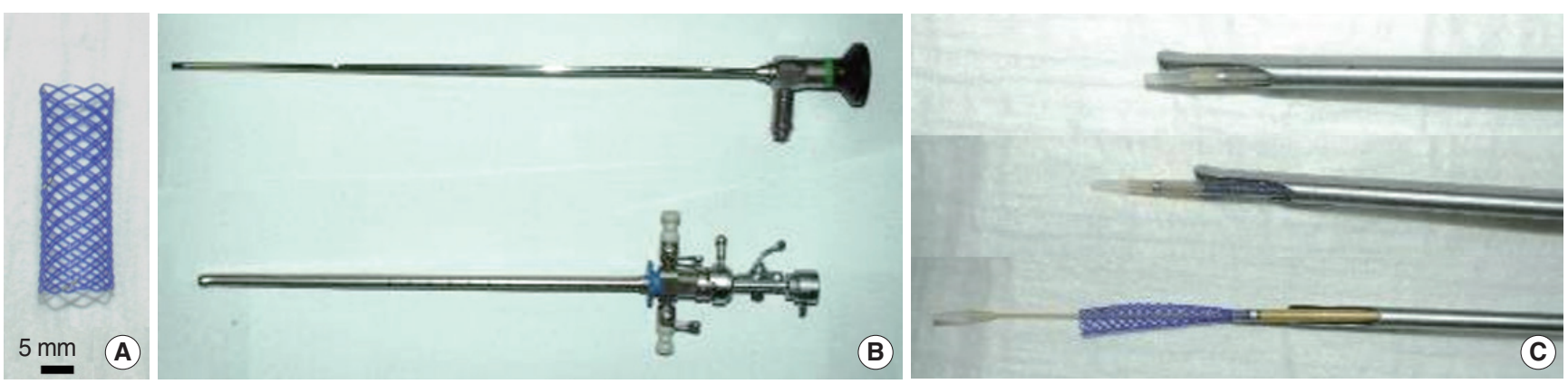

Fig. 1. Polydioxanone (PDO) tracheal stent and endoscopic delivery tools. (A) PDO stent $(8 \times 30 \mathrm{~mm})$. (B, C) Transoral tracheal stent delivery system.

Conventional non-biodegradable stents have been used to secure airway patency and to avoid invasive surgical procedures. The first-generation tracheal stent was made of silicone, but migration of the stent and mucociliary clearance disturbance were common problems [4-6]. Moreover, a mortality rate of 32.5\% was reported in a study of pediatric patients [7]. A mesh-type metallic stent allows migration of mucosal cells and regeneration of the airway epithelium, thereby facilitating mucociliary clearance [4]. However, restenosis by granulation tissue has been noted following metallic stent placement, resulting in tracheal narrowing and necessitating stent removal $[8,9]$. In addition, metallic stent placement is associated with high mortality; in one study, three out of nine children died of tracheal hemorrhage [10].

Biodegradable stents may be effective for children because their favorable degradation properties may reduce the trauma and adverse effects of stent removal. These studies only included a small number of cases, and the few animal studies that have been conducted did not use rigorous models, so the natural course of these stents has not yet been fully elucidated [11-14].

Polydioxanone (PDO) is widely used for clinical bioabsorbable sutures because its biocompatibility enables cell adhesion and infiltration [15-18]. PDO monofilaments have been used to create a mesh-type stent [19], which has been employed in pediatric patients with airway problems. Although concerns have been raised that degraded stent fragments could induce inflammation and obstruction of the distal bronchioles or alveoli, no previous study has investigated the safety of this biodegradable stent $[12,20,21]$.

For this reason, we designed an in vivo experiment to closely

\section{H I G G H L L I}

- An animal model that closely reflected the pathology of pediatric tracheomalacia was developed.

- A biodegradable polydioxanone stent prolonged the survival of tracheomalacia animals.

- The outcomes of stents observed in the tracheomalacia-model rabbits differed from those in normal rabbits. mirror the treatment of tracheomalacia in pediatric patients. We used rabbits, which have similar-sized tracheas to human infants [22], and employed the same stenting procedure as in these patients. We attempted to answer four key questions: is this biodegradable PDO stent safe? Could this biodegradable PDO stent be accurately deployed endoscopically to control tracheomalacia? How long do the beneficial effects of the stent last? Does the PDO stent have detrimental effects on the tracheal mucosa, and if so, are these reversible after stent degradation?

\section{MATERIALS AND METHODS}

A stent (Taewoong Medical Co., Gimpo, Korea) of 8-mm diameter and 30-mm length (Fig. 1) was manufactured using commercially available PDO monofilament (Meta Biomed Co., Cheongju, Korea). An in vitro degradation test of the PDO stent was performed (Supplementary Material 1).

A total of 29 New Zealand white (NZW) rabbits (male, 3.0$3.5 \mathrm{~kg}$ ) were used in this study. The flowchart of the experiment for all 29 rabbits is shown in Fig. 2. In all, 13 rabbits were used to evaluate the biocompatibility of the PDO stent in a normal trachea and 16 were used as tracheomalacia animal models. Of the 16 , tracheomalacia was successfully established in 12 rabbits. All animal procedures were approved by the Institutional Animal Care and Use Committee of Seoul National University Hospital and animals were maintained in an Association for Assessment and Accreditation of Laboratory Animal Care International-accredited facility (\#001169) in accordance with the National Research Council's 2010 Guide for the Care and Use of Laboratory Animals, 8th edition.

The surgical procedure was performed according to a previously described protocol with minor modifications (Fig. 3, Supplementary Material 2) [23]. Tracheomalacia models were created in which the anterior tracheal cartilage was resected for a length of $2 \mathrm{~cm}$ from the third tracheal ring. Collapse of the tracheal lumen during breathing after anterior tracheal cartilage resection is demonstrated in Supplementary Video 1. Previously, severe tracheomalacia was defined as the anteroposterior collapse of 


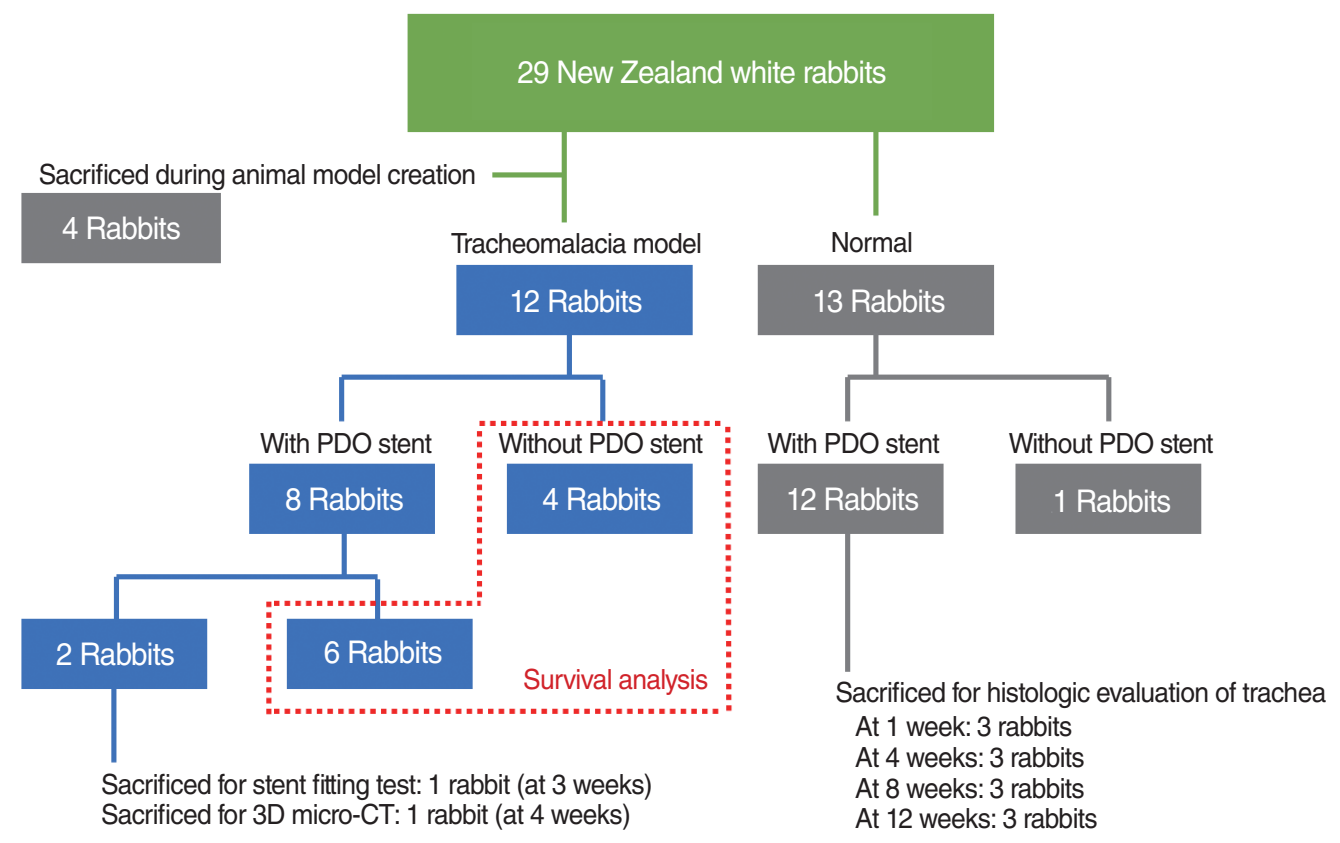

Fig. 2. Flowchart of animal experiments. The number of rabbits used in each experiment, and the time and purpose of their sacrifice are shown. PDO, polydioxanone; 3D, three-dimensional; micro-CT, micro-computed tomography.
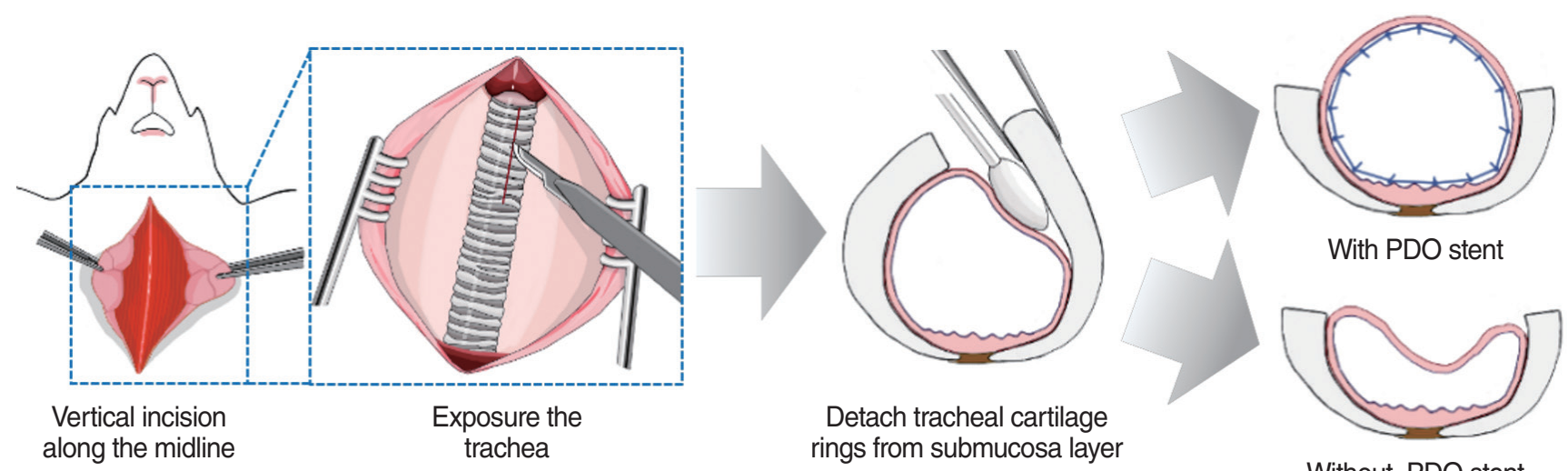

Without PDO stent

Fig. 3. Schematic representation showing the process of how to develop the tracheomalacia rabbit model. PDO, polydioxanone.

the tracheal lumen during respiration of greater than 75\% [24]. All tracheomalacia model rabbits met the condition of severe tracheomalacia immediately after cartilage resection. Every day for a week, each animal was injected with $2 \mathrm{mg} / \mathrm{kg}$ gentamicin (Daesung Microbiological Labs. Co., Seoul, Korea) and $15 \mathrm{mg} / \mathrm{kg}$ Sulpyrine (Daesung Microbiological Labs. Co.). Animals were monitored daily for clinical signs such as weight changes, cough, sputum, wheezing, and dyspnea. An animal was to be euthanized immediately when it exhibited a $20 \%$ weight loss or symptoms of severe respiratory distress. According to the criteria, four of six stented tracheomalacia rabbits were euthanized due to respiratory distress. The two others died before severe respiratory symptoms developed. The four without-stent tracheomalacia rabbits had moderate to severe respiratory distress immediately after creation and died without further exacerbations.
All devices including the PDO stent were sterilized using ethylene oxide gas at $50^{\circ} \mathrm{C}$ for 16 hours before use. Each animal was anesthetized using an intramuscular injection containing $0.83 \mathrm{~mL} / \mathrm{kg}$ xylazine (Rompun, Bayer Korea, Seoul, Korea) and $0.83 \mathrm{~mL} / \mathrm{kg}$ ketamine (Yuhan Co., Seoul, Korea) and placed in the supine position. The stent was manually loaded into a proximal release delivery system (Taewoong Medical Co.) and endoscope-guided stent insertion was conducted. Balloon dilation catheters (Inflated balloon OD 6-7-8 mm; CRE Balloon Dilation Catheters, Boston Scientific, Marlborough, MA, USA) were used for stent expansion (Fig. 1), which was performed at a pressure of $10 \mathrm{~atm}$ (8-mm diameter) for 5 seconds (Supplementary Video 2). Clinical signs such as weight changes, cough, sputum, wheezing, and dyspnea were monitored daily. Animals were euthanized when they exhibited $20 \%$ weight loss or symptoms of severe 

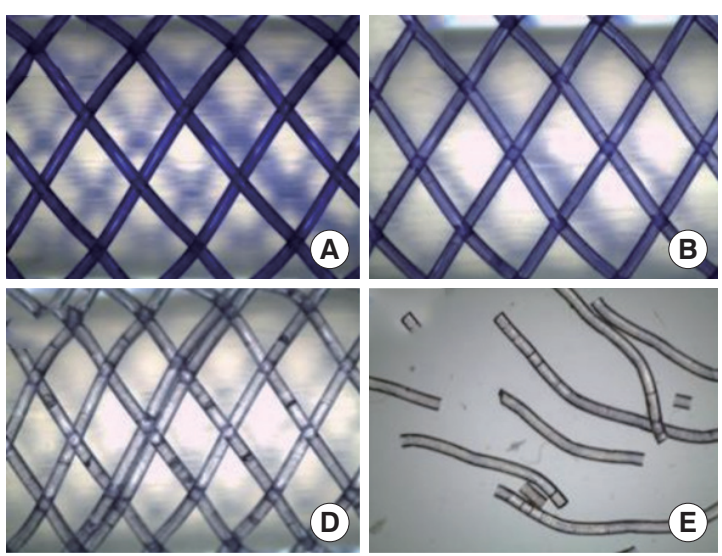
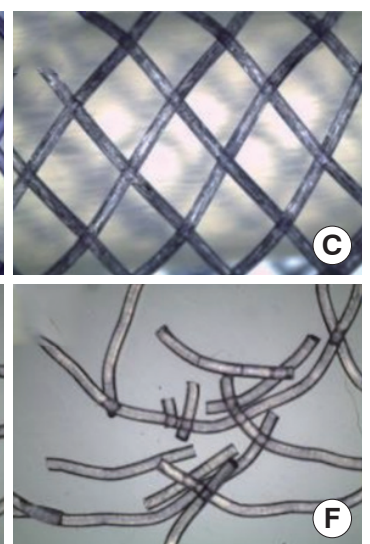

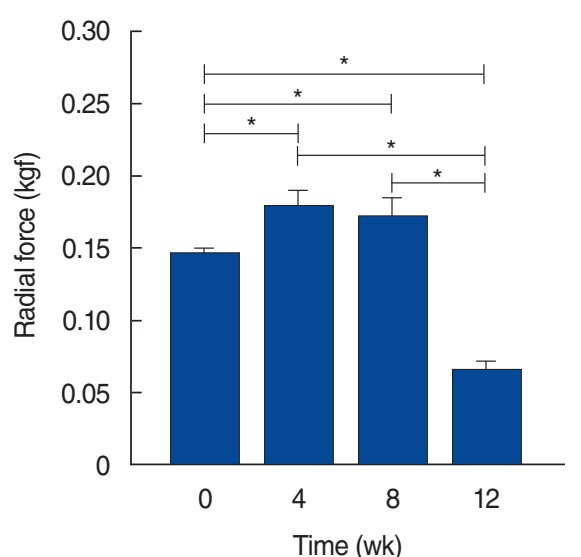

Fig. 4. Microscopic images of in vitro degradation of the polydioxanone (PDO) stent at (A) 0, (B) 4, (C) 8, (D) 12, (E) 16, and (F) 20 weeks following stent placement (magnification, $\times 28)$. (G) Mechanical strength of PDO stent after degradation in vitro $\left({ }^{*} P<0.001\right)$.
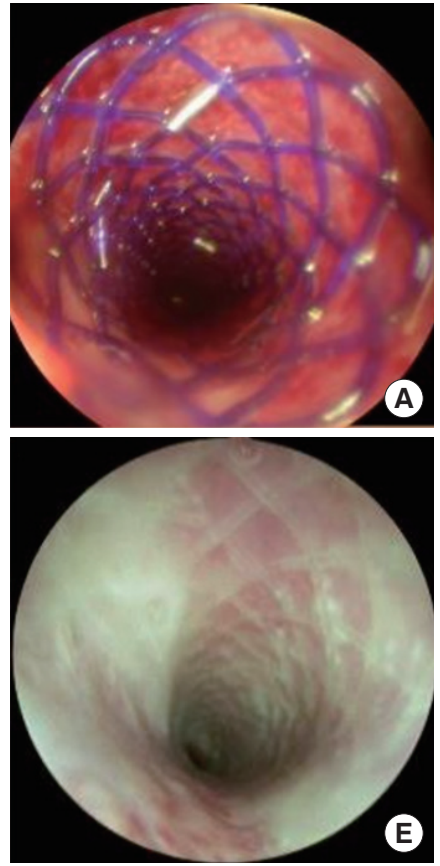
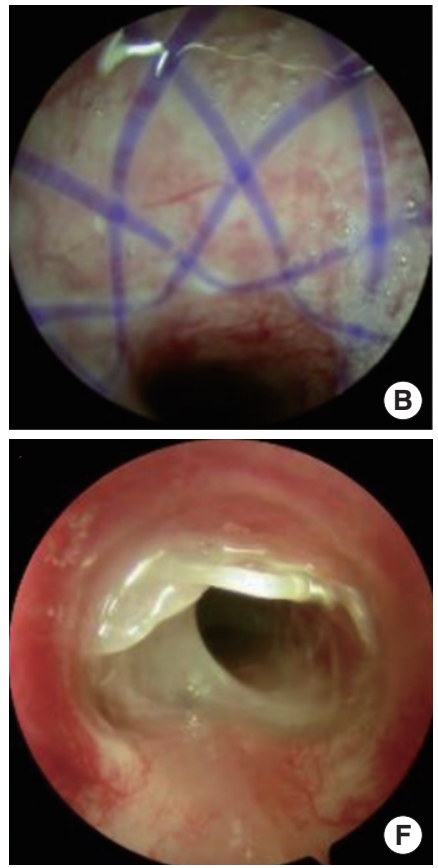
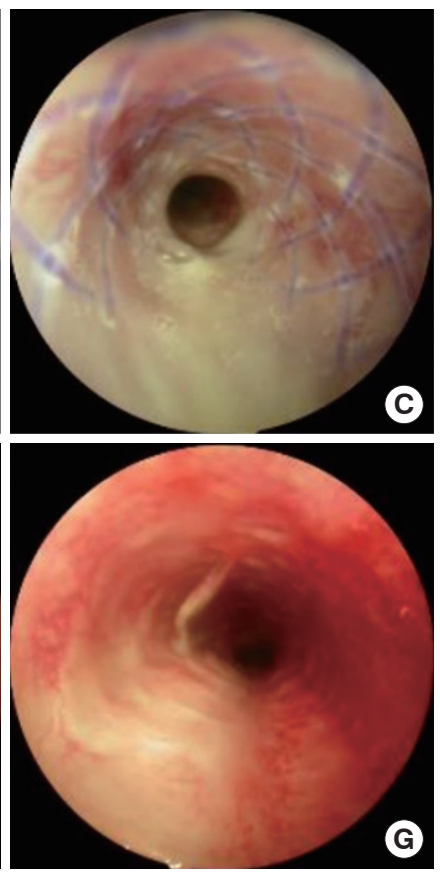
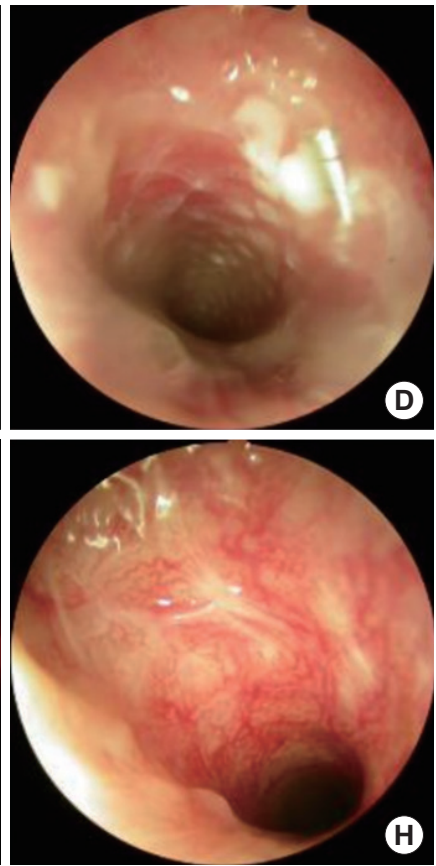

Fig. 5. Bronchoscopy images taken (A) immediately after stenting and (B) 1, (C) 2, (D) 4, (E) 6, (F) 8, (G) 10, and (H) 12 weeks after stenting. (G) At 10 weeks, the stent had decomposed, and debris was observed. $(\mathrm{H})$ At 12 weeks, no material was observed inside the trachea.

respiratory distress.

To evaluate the biodegradation and biocompatibility of the PDO stent and recovery of the epithelium after stenting in the normal NZW rabbits, the animals were anesthetized with an intramuscular injection of $0.83 \mathrm{~mL} / \mathrm{kg}$ xylazine and $0.83 \mathrm{~mL} / \mathrm{kg}$ ketamine $1,2,4,6,8,10$, and 12 weeks after stent placement. Then the airways of the animals were observed using an endoscopic system. After 1, 4, 8, and 12 weeks from the stenting, rabbits were sacrificed and the full length of the trachea containing the PDO stent and the lungs were harvested for morphology and histological evaluation. The tissues were histologically evaluated by hematoxylin and eosin, Masson's trichrome, Alcian blue,TUNEL (terminal deoxynucleotidyl transferase dUTP nick end labeling), and immunohistochemical staining (Supplementary Material 3).

Statistical significance was determined by one-way analysis of variance using SigmaPlot 12.0 (Systat Software Inc., San Jose, CA, USA) and $P<0.05$ was considered statistically significant.

\section{RESULTS}

Stents in the normal trachea

The in vitro degradation test results are presented in Fig. 4 and Supplementary Material 1. In the in vivo study, degradation of 
the PDO stent was not observed until 8 weeks after stent placement, and obvious fractures of the stent as a result of degradation were observed after 10 weeks (Fig. 5). Nonetheless, no obstruction of the airway by fragments of the degraded PDO stent was observed. The fragmented stent gradually disappeared at the insertion site 12 weeks after stent placement and did not cause tracheal granulation. Although we did not definitively confirm whether fragments of the degraded PDO stent were expectorated or fell into the lungs, we considered the possibility that some fragments may have entered the lungs. Hence, we examined the distal airways and alveoli; no abnormalities in the distal airway were detected, nor was mucosal damage or inflammation observed (Supplementary Fig. 1).

We evaluated changes in the mucosa of the airway epithelium after stenting by histological staining at 1, 4, 8, and 12 weeks. As shown in Fig. 6 (the corresponding biopsy sites were shown in Supplementary Fig. 2) and Supplementary Fig. 3, 1 week after stent placement, the airway epithelium located under a strand of the PDO stent exhibited tissue damage, as shown by submucosal fibrotic collagen deposition. Moreover, the compressed epithelium had lost its CK5-positive basal layer and intrinsic respiratory cells such as mucin-secreting goblet cells and ciliated cells ( $\beta$-tubulin-positive cells). However, between 4 and 12 weeks after stenting, this damaged tissue was regenerated. In detail, although granulation tissue was observed beside a strand of the stent that compressed the epithelium at 4 weeks, the thickness of this granulation tissue decreased at 8 weeks and the thickness of the epithelium recovered to a level comparable to the native epithelium at 12 weeks. In addition, the amount of deposited collagen gradually decreased and exhibited intrinsic alignment. At 4 weeks, CK5-positive cells, which could enhance cell proliferation and tissue regeneration at the basal level, began to be
$H \& E$
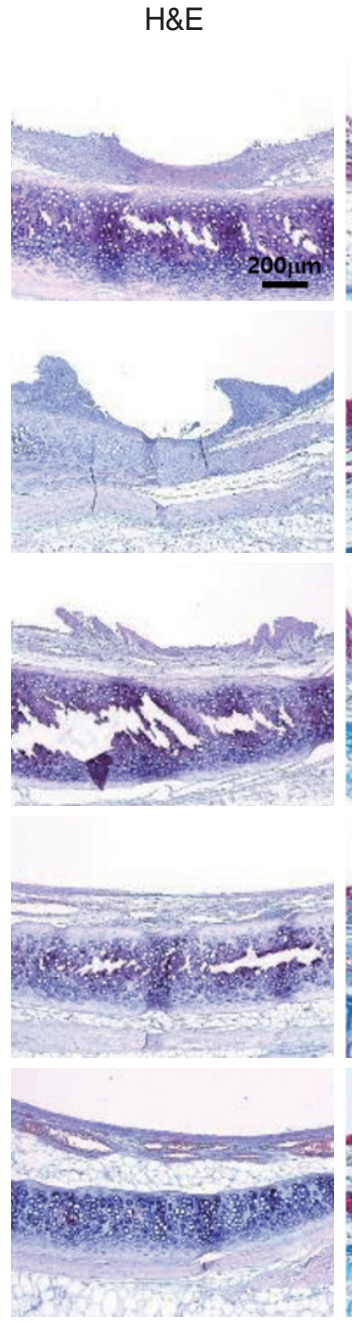

Masson's trichrome
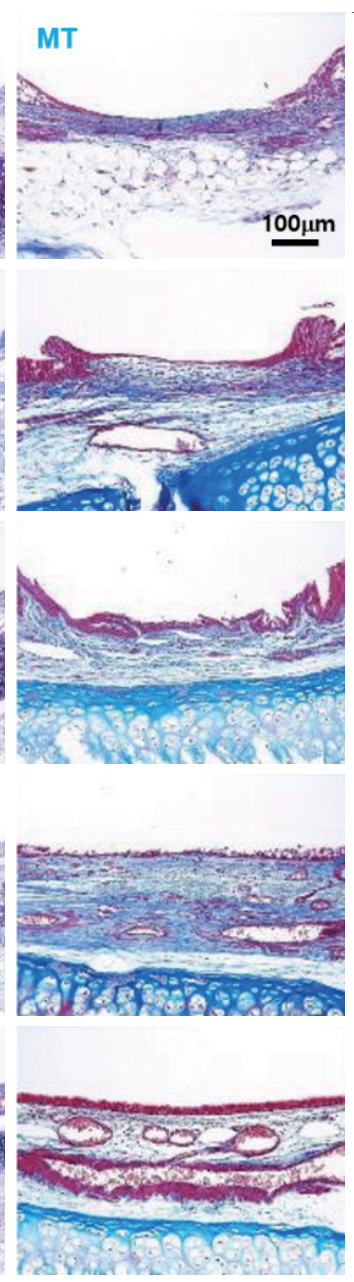

Anti-CK5
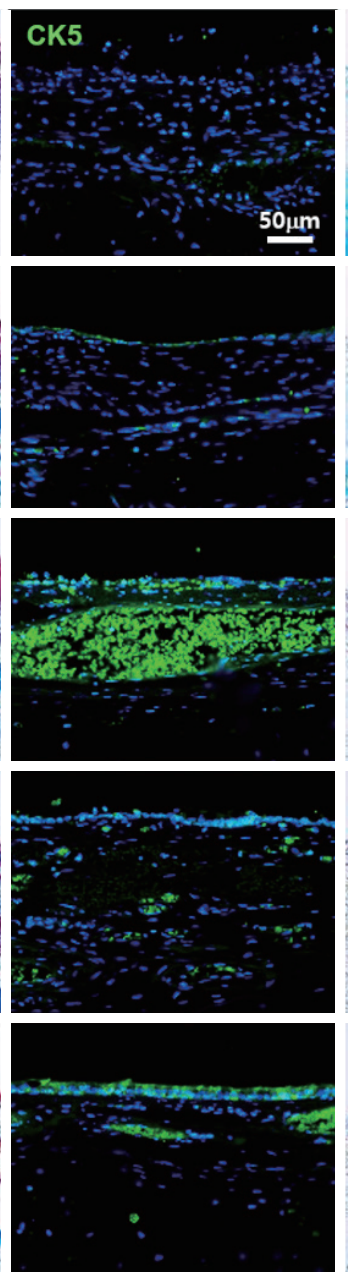

Alcian blue
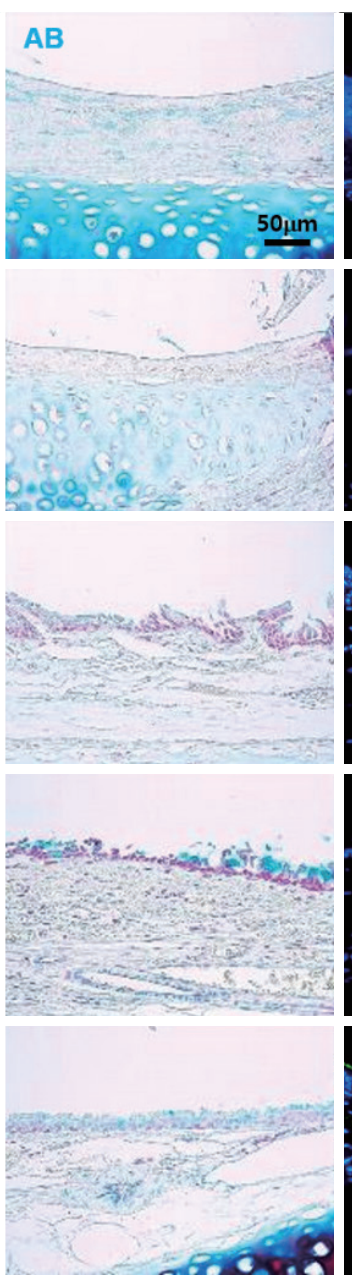

Anti- $\beta$-tubulin
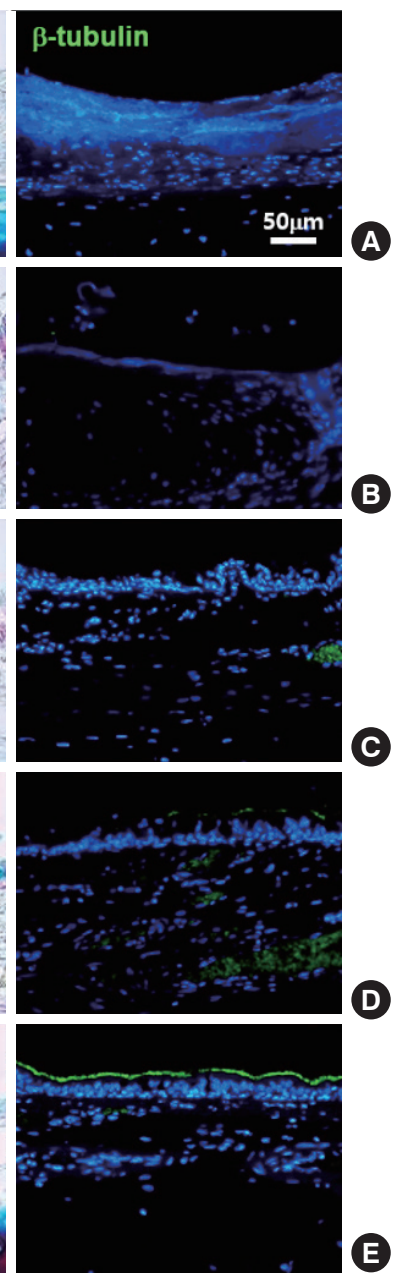

Fig. 6. Histological evaluation of the airway epithelium of the normal trachea at (A) 1, (B) 4, (C) 8, and (D) 12 weeks after stenting and (E) no stenting. Collagen is stained blue by Masson's trichrome. Mucin is stained blue by Alcian blue. Nuclei are stained blue by 4',6-diamidino2-phenylindole (DAPI) in immunohistochemistry against CK5 and $\beta$-tubulin. The location from which each tissue was sampled is marked in red in Supplementary Fig. 2. 
observed and the number of CK5-positive cells significantly increased at 8 weeks. Moreover, the expression of goblet cell-secreted mucin was confirmed at 8 weeks, representing functional recovery of the airway epithelium. $\beta$-tubulin expression was observed in the regenerated epithelium, indicating the regeneration of ciliated cells, although the epithelium was not completely covered by these regenerated ciliated cells at 12 weeks.

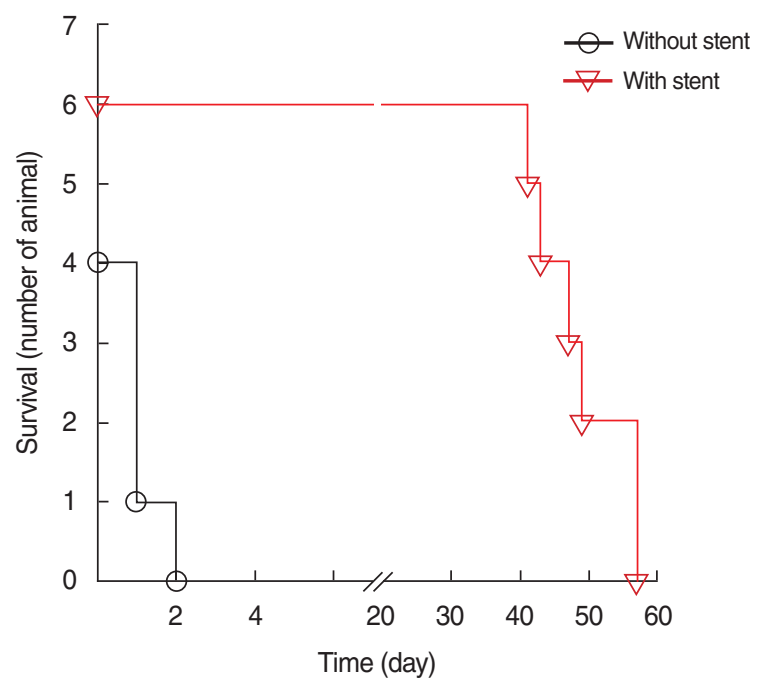

Fig. 7. Survival of tracheomalacia animals with and without stents. All tracheomalacia rabbits without stents died within 2 days. The survival duration of rabbits with stents was significantly longer than rabbits without stents ( $49.0 \pm 6.8$ vs. $1.0 \pm 0.8$ days, $P=0.001)$.

\section{Stents in the animal model}

A total of 16 rabbits were used to create the animal tracheomalacia model. This procedure failed four times during tracheal cartilage and submucosa layer dissection, so the overall success rate was $75.0 \%(12 / 16)$. Of the 12 tracheomalacia-model rabbits, eight were stented and four were not stented. Two of the eight stented rabbits were sacrificed: one for tracheal three-dimensional reconstruction using micro-computed tomography (micro-CT) at 4 weeks and the other for a stent size fitting test at 3 weeks. Finally, six tracheomalacia rabbits with stents and four without stents were used to analyze the survival rate. All the tracheomalacia rabbits without stents died within 2 days (three of them on the day of the procedure), and the average survival duration of this group was $1.0 \pm 0.8$ days. The average survival duration of the six rabbits with stents was $49.0 \pm 6.8$ days. Therefore, the PDO stent showed a significant survival benefit $(P=0.001)$ (Fig. 7).

Immediately after tracheomalacia induction, the airway collapsed during the exhalant phase of respiration (Fig. 8). After stent placement, the airway did not collapse during respiration. Degradation of the PDO stent was observed starting at 4 weeks. As in the normal trachea, there was no obstruction of the airway by fragments of the degraded PDO stent. At 7 weeks, PDO stent degradation and granulation tissue were identified. Because the longest survival duration of the tracheomalacia rabbits was less than 8 weeks, no further observations were possible.

We reconstructed micro-CT images to confirm the maintenance of airway patency in four rabbits (Fig. 9); one set of images was taken in in a rabbit with no stent, one was taken in a stented
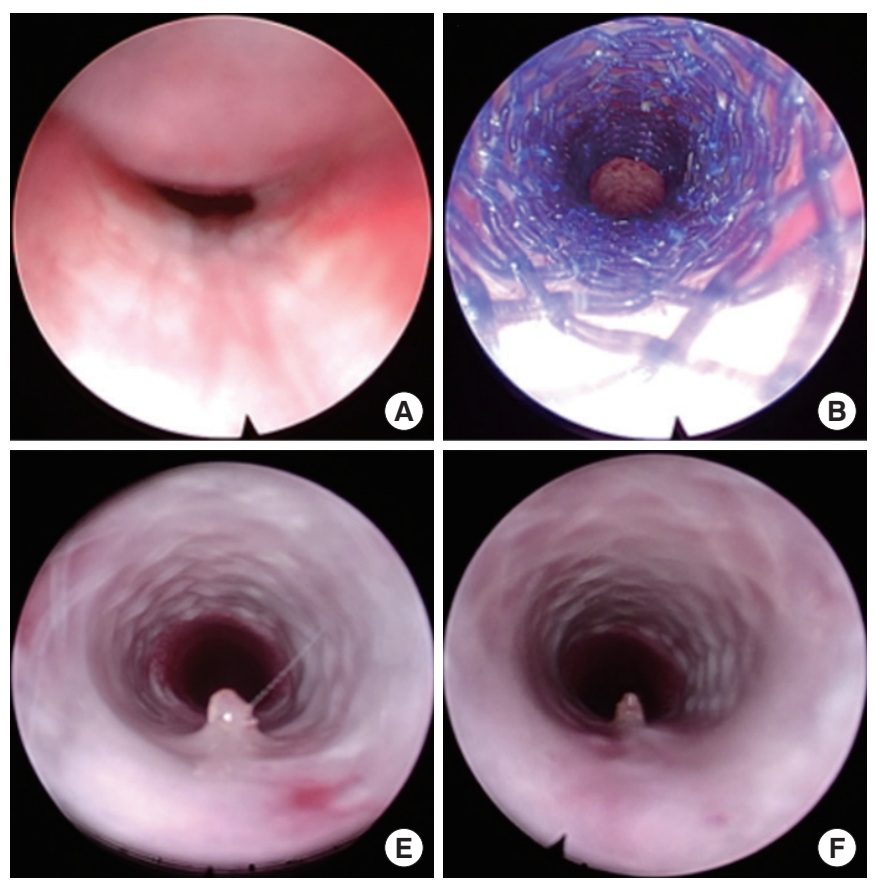
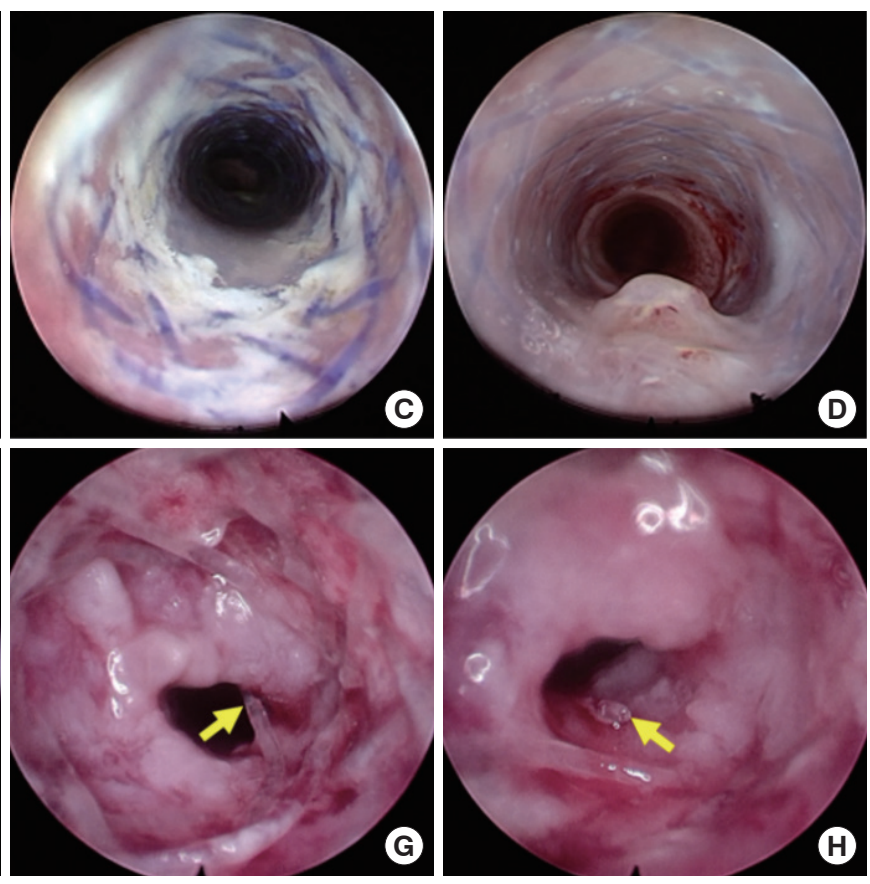

Fig. 8. Serial endoscopic images of the airway (A) after creating tracheomalacia, (B) after stenting, and (C) 1, (D) 2, (E) 4, (F) 6, and (G, H; postmortem) 7 weeks after stenting. Yellow arrows $(\mathrm{G}, \mathrm{H})$ show a degraded polydioxanone stent. 

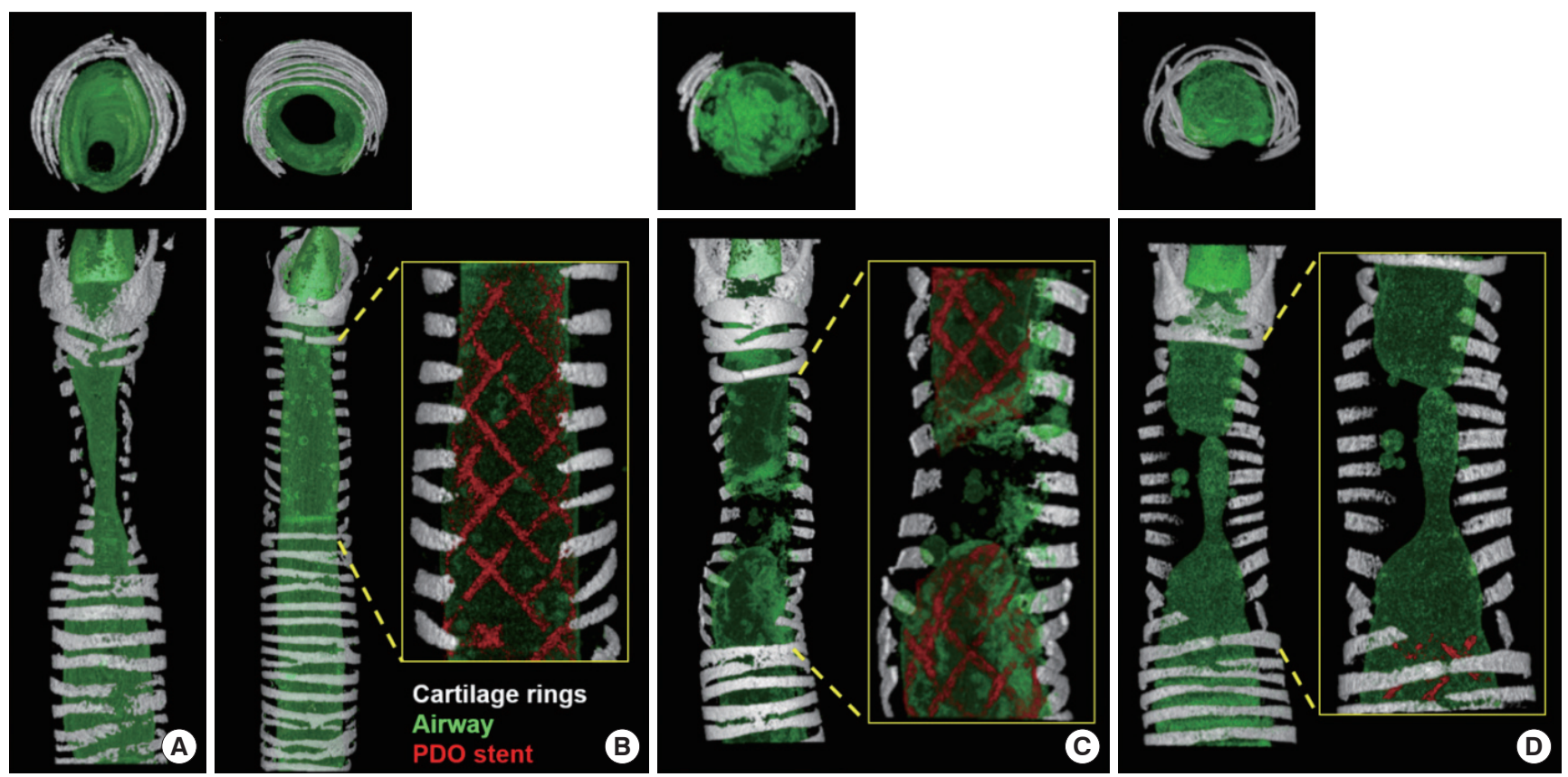

Fig. 9. Reconstructed micro-computed tomography images of the trachea $(A)$ without a stent 1 day after the induction of tracheomalacia and (B-D) with a stent at (B) 4, (C) 7, and (D) 8 weeks after stenting. The upper and lower panels represent axial and longitudinal views, respectively. PDO, polydioxanone.

rabbit before sacrifice, and the other two were taken just after death in the stented rabbits. The three-dimensional reconstruction showed tracheal airway narrowing in the rabbits without a stent, but the stent overcame this airway narrowing and maintained the patency of the airway. However, at 7 or 8 weeks, discontinuity of the stent and airway collapse were observed. The middle portion of the stent was the first to collapse. At week 7, the stent remained at both ends of the tracheomalacia segment, but at week 8 only a small portion of the stent remained in the normal tissue outside of the tracheomalacia segment.

\section{DISCUSSION}

Airway stents for tracheomalacia are gaining attention because their placement is noninvasive [5]. Existing non-degradable stents are made of plastic (e.g., silicone) or metal (e.g., stainless steel and nickel-titanium) [5]. However, these are associated with migration, granulation, and mucous plugging, and may necessitate risk-associated stent removal [9]. Although the long-term efficacy of metallic stents has been demonstrated [8], tracheomalacia mainly occurs in growing children; therefore, it is important to consider the issue of stent removal and replacement with a larger-diameter stent due to the increase in the internal diameter of the trachea [25]. However, it is difficult to remove a metallic stent once it is mucosalized.

In this respect, biodegradable stents have both an advantage and a disadvantage: the advantage is that extraction of the de- vice is unnecessary [26], while the disadvantage is that the short maintenance period of the stent requires repeated operations. There have been individual reports of PDO stent use in tracheomalacia patients $[11,20]$; however, its safety has not been sufficiently established for universal clinical application. One concern relates to the disassembled stent fragments, since pediatric patients have a narrow internal bronchial diameter and may exhibit difficulties in expectoration. Indeed, studies have suggested a risk of obstruction due to large stent fragments $[12,20]$. In this regard, more data regarding the safety and efficacy of this PDO stent are needed from animal studies before clinical trials in pediatric patients can be conducted.

Our study had two significant strengths compared to previous animal studies. First, the duration of stent maintenance is different between normal tracheas and those affected by tracheomalacia, making it necessary to evaluate the safety and efficacy of stents in animal models that accurately represent tracheomalacia. In our study, an appropriate tracheomalacia model was devised. Because pediatric patients suffering from tracheomalacia are the main target group for the stent, we chose NZW rabbits because the size of their trachea is similar to that of human infants. Second, the same endoscopic stenting procedure used in clinical practice was applied in this animal model. To the best of our knowledge, this is the first study to deploy biodegradable stents guided by an endoscope in a tracheomalacia model using the same procedure as in patients. In most previous studies, the trachea was accessed externally with open neck surgery, and the majority did not use a tracheomalacia model [26-31]. Novotny 
et al. [13] employed fluoroscopic-guided insertion, although their study was performed in normal rabbits.

In a previous study of the degradation of PDO in an environment with a similar temperature and $\mathrm{pH}$ to the human body, a $3 \%$ decrease in stent weight was noted after 10 weeks, but only $13.44 \%$ of its initial strength remained [32,33]. In our in vitro study, the PDO stent was morphologically stable at 12 weeks and degraded at 16 weeks. However, the radial force of the stent significantly decreased at 12 weeks. In our in vivo study, all PDO stent particles disappeared at 12 weeks in the normal trachea. This stent degradation time in the normal trachea is consistent with previous in vitro and in vivo studies [13,32].

In the tracheomalacia model, stridor began to develop after about 6 weeks and stented animals died at around 7 weeks. Likewise, in the endoscopic study, collapse of the stent was observed around 7 weeks. To the best of our knowledge, this is the first study to demonstrate a shortened stent degradation time in a tracheomalacia model. Further studies are required to elucidate the mechanism underlying this finding, but it may have been due to the absence of tracheal rings. As a result, when rotating or flexing the neck, external forces pressing on the trachea are repeatedly applied to the stent, accelerating stent decomposition. This hypothesis is supported by the reconstructed micro-CT images. At 7 weeks, the stent in the tracheomalacia segment had disintegrated from the center. At 8 weeks, the stent remained under the normal cartilage rings, which may have been protected from external forces. As such, the effects of vascular compression, a cause of tracheomalacia, on the stability of the PDO stent should be evaluated, since it may be affected by the pulsatile compressive force exerted by the vessels. Prior to clinical trials, the effects of external compression on the duration of the effects of the PDO stent should also be evaluated.

Compression during stent expansion may induce damage to the airway epithelium as the cells are squashed, torn, and crushed. This can disrupt the structural integrity and biological functions of epithelial cells [34]. We observed epithelial cell death and inflammation at 1 week after stent placement in the histologic evaluation. However, at 4 weeks after stenting, the number of dead and inflammatory cells had decreased, and the compressed extracellular matrix had recovered its thickness and alignment. Although fragments of the degraded PDO stent were observed at 10 weeks, we consider it likely that degradation occurred gradually before then and the pressure exerted by the inserted PDO stent weakened $[32,33]$. Hence, we speculate that this reduction in compression facilitated the regeneration of the damaged epithelium. Furthermore, CK5-positive cells had regenerated at the basal layer at 4 weeks, initiating regeneration of intrinsic respiratory function [35]. Moreover, at 12 weeks, mucin and $\beta$-tubulin expression was identified on the regenerated airway epithelium, representing the recovery of mucociliary clearance.

In the bronchoscopic evaluation of the normal rabbits, fragmented stents were observed at the insertion sites, but airway obstruction by the fragmented particles was not noted. In addition, no abnormalities were observed in the bronchioles and alveoli. It appeared that the stents gradually dissolved during degradation and were not broken into smaller particles. Similarly, in the tracheomalacia model, no evidence of particles falling into the distal airways after stent degradation was observed.

Stent collapse preceded the deaths of all tracheomalacia model rabbits. Although we did not conduct re-stenting at this point, we identified some questions that should be addressed. First, the timing of re-stenting needs to be investigated. In the tracheomalacia model, airway symptoms such as stridor were observed starting at 6 weeks, whereas stent collapse was not observed until 7 weeks. The second consideration is whether to remove the existing stent fragments when re-stenting is performed. We did not observe migration or dislocation of fragments after stent collapse; however, re-stenting without removing residual fragments may push those fragments to the distal airways, causing occlusion. Conversely, luminal damage or perforation may occur during fragment removal. The third consideration is whether stentinduced fibrosis could result in more robust mechanical properties of the malacic segment that would protect against collapse. In our study, 7 weeks was not enough to observe this possibility. Further research is needed to confirm the timing and method of re-stenting.

The study had some limitations that should be noted. First, it was difficult to make an animal model. This initial experiment had a $75 \%(12 / 16)$ success rate, and it should be performed by ENT doctors with experience using micro instruments. Second, our model had limited versatility. Removal of the anterior tracheal cartilage does not fully reflect the mechanism of all types of tracheomalacia, particularly posterior tracheomalacia. Third, as the study was designed for in-depth examination of a PDO stent, a comparison to existing metallic or silicone stents was not performed.A further comparative study is needed.

We demonstrated the successful development of a rabbit tracheomalacia model. To the best of our knowledge, this is the first study to successfully conduct endoscope-guided stent placement in normal and tracheomalacia rabbits. Although the PDO stent caused mucosal changes in the normal trachea, these were reversible. Furthermore, the stent did not obstruct the distal bronchus. In the tracheomalacia model, the PDO stent promoted survival. Notably, however, degradation of the stent occurred faster in these tracheomalacia animals; the stent decomposed after 7 weeks and all animals died. Further studies are therefore required to elucidate the optimal method and timing of re-stenting after stent degradation.

\section{CONFLICT OF INTEREST}

Among the authors of this paper, Sang Min Joo, Yeo Jin Jun, and Kil Won Son are employees of Taewoong Medical Co., Ltd., the 
company that manufactures the stent. They contributed to the development of the stent and instrumentation. There are no other potential conflicts of interest relevant to this article.

\section{ACKNOWLEDGMENTS}

This research was supported by a grant of the Korea Health Technology R\&D Project through the Korea Health Industry Development Institute, funded by the Ministry of Health \& Welfare, Republic of Korea (grant numbers HI18C1174 and HI14C1277), by the Bio \& Medical Technology Development Program of the National Research Foundation (NRF) funded by the Korean government (MSIT) (grant number 2019M3A9H1103617), and by Seoul National University Research Fund (grant number 0420193030).

\section{ORCID}

$\begin{array}{ll}\text { Ji Suk Choi } & \text { https://orcid.org/0000-0002-7127-1858 } \\ \text { Jungirl Seok } & \text { https://orcid.org/0000-0001-6473-6015 } \\ \text { Min Rye Eom } & \text { https://orcid.org/0000-0001-8037-2522 } \\ \text { Eungee Jung } & \text { https://orcid.org/0000-0002-1816-8026 } \\ \text { Su A Park } & \mathrm{https://orcid.org/0000-0001-5878-8054} \\ \text { Sang Min Joo } & \mathrm{https://orcid.org/0000-0001-7886-0973} \\ \text { Yeo Jin Jun } & \mathrm{https://orcid.org/0000-0001-6039-0926} \\ \text { KilWon Son } & \mathrm{https://orcid.org/0000-0002-2412-6666} \\ \text { Seong Keun Kwon } & \mathrm{https://orcid.org/0000-0001-9218-7666}\end{array}$

\section{AUTHOR CONTRIBUTIONS}

Conceptualization: SAP, SMJ, SKK. Data curation: JSC, JS, MRE, EJ, SAP, SMJ, YJJ, KWS. Formal analysis: JSC, SAP, SMJ. Funding acquisition: SKK. Methodology: JSC, SMJ, SKK. Project administration: SKK. Visualization: JSC, JS. Writing-original draft: JSC, JS. Writing-review \& editing: JSC, JS, SKK.

\section{SUPPLEMENTARY MATERIALS}

Supplementary materials can be found via https://oi.org/10.21053/ ceo.2020.01627.

\section{REFERENCES}

1. Carden KA, Boiselle PM, Waltz DA, Ernst A. Tracheomalacia and tracheobronchomalacia in children and adults: an in-depth review. Chest. 2005 Mar;127(3):984-1005.

2. Boogaard R, Huijsmans SH, Pijnenburg MW,Tiddens HA, de Jongste JC, Merkus PJ. Tracheomalacia and bronchomalacia in children: incidence and patient characteristics. Chest. 2005 Nov;128(5):3391-7.
3. McNamara VM, Crabbe DC. Tracheomalacia. Paediatr Respir Rev. 2004 Jun;5(2):147-54.

4. SaitoY, Imamura H. Airway stenting. Surg Today. 2005;35(4):265-70.

5. Zakaluzny SA, Lane JD, Mair EA. Complications of tracheobronchial airway stents. Otolaryngol Head Neck Surg. 2003 Apr;128(4): 478-88.

6. Korraa E, Madkour A, Todary A, Wagieh K. Evaluation of bronchoscopic placement of tracheobronchial silicone stents: an Ain Shams University Hospital experience. Egypt J Bronchol. 2014;8(1):38-43.

7. Jacobs JP, Quintessenza JA, Botero LM, van Gelder HM, Giroud JM, Elliott MJ, et al. The role of airway stents in the management of pediatric tracheal, carinal, and bronchial disease. Eur J Cardiothorac Surg. 2000 Nov;18(5):505-12.

8. Saad CP, Murthy S, Krizmanich G, Mehta AC. Self-expandable metallic airway stents and flexible bronchoscopy: long-term outcomes analysis. Chest. 2003 Nov;124(5):1993-9.

9. Lunn W, Feller-Kopman D, Wahidi M, Ashiku S, Thurer R, Ernst A. Endoscopic removal of metallic airway stents. Chest. 2005 Jun; 127(6):2106-12.

10. Geller KA, Wells WJ, Koempel JA, St John MA. Use of the Palmaz stent in the treatment of severe tracheomalacia. Ann Otol Rhinol Laryngol. 2004 Aug;113(8):641-7.

11. Vondrys D, Elliott MJ, McLaren CA, Noctor C, Roebuck DJ. First experience with biodegradable airway stents in children. Ann Thorac Surg. 2011 Nov;92(5):1870-4.

12. Sztano B, Kiss G, Marai K, Racz G, Szegesdi I, Racz K, et al. Biodegradable airway stents infants: potential life-threatening pitfalls. Int J Pediatr Otorhinolaryngol. 2016 Dec;91:86-89.

13. Novotny L, Crha M, Rauser P, Hep A, Misik J, Necas A, et al. Novel biodegradable polydioxanone stents in a rabbit airway model. JThorac Cardiovasc Surg. 2012 Feb;143(2):437-44.

14. Kawahara I, Ono S, Maeda K. Biodegradable polydioxanone stent as a new treatment strategy for tracheal stenosis in a rabbit model. J Pediatr Surg. 2016 Dec;51(12):1967-71.

15. Zamiri P, Kuang Y, Sharma U, Ng TF, Busold RH, Rago AP, et al.The biocompatibility of rapidly degrading polymeric stents in porcine carotid arteries. Biomaterials. 2010 Nov;31(31):7847-55.

16. Zhou X, Pan Y, Liu R, Luo X, Zeng X, Zhi D, et al. Biocompatibility and biodegradation properties of polycaprolactone/polydioxanone composite scaffolds prepared by blend or co-electrospinning. J Bioact Compat Polym. 2019;34(2):115-30.

17. Yanhui L, Ruitao J, Mian W, Shaoju F, Peihua Z. Degradation and biocompatibility behaviors of fully covered biodegradable polydioxanone biliary stent for human body. Ind Textila. 2019;70(5):393-7.

18. Hakimi O, Murphy R, Stachewicz U, Hislop S, Carr AJ. An electrospun polydioxanone patch for the localisation of biological therapies during tendon repair. Eur Cell Mater. 2012 Oct;24:344-57.

19. Li G, LiY, Lan P, Li J, Zhao Z, He X, et al. Biodegradable weft-knitted intestinal stents: fabrication and physical changes investigation in vitro degradation. J Biomed Mater Res A. 2014 Apr;102(4):982-90.

20. Stehlik L, Hytych V, Letackova J, Kubena P,Vasakova M. Biodegradable polydioxanone stents in the treatment of adult patients with tracheal narrowing. BMC Pulm Med. 2015 Dec;15:164.

21. Vondrys D, Anton-Pacheco Sanchez J. Letter to the editor regarding "Biodegradable airway stents in infants: potential life-threatening pitfalls". Int J Pediatr Otorhinolaryngol. 2017 Jul;98:174.

22. Miller FR, Guay ME, Bauer T, Tucker HM. Long-term flap tracheostomy in a pediatric animal model. Arch Otolaryngol Head Neck Surg. 1995 Jul;121(7):743-8.

23. Gorostidi F, Courbon C, Burki M, Reinhard A, Sandu K. Extraluminal biodegradable splint to treat upper airway anterior malacia: a preclinical proof of principle. Laryngoscope. 2018 Feb;128(2):E53-8.

24. Filler RM, Messineo A, Vinograd I. Severe tracheomalacia associated with esophageal atresia: results of surgical treatment. J Pediatr Surg. 
1992 Aug;27(8):1136-40.

25. Hysinger EB, Panitch HB. Paediatric tracheomalacia. Paediatr Respir Rev. 2016 Jan;17:9-15.

26. Saito Y, Minami K, Kobayashi M, Nakao Y, Omiya H, Imamura H, et al. New tubular bioabsorbable knitted airway stent: biocompatibility and mechanical strength. JThorac Cardiovasc Surg. 2002 Jan;123(1): 161-7.

27. Liu KS, Liu YH, Peng YJ, Liu SJ. Experimental absorbable stent permits airway remodeling. JThorac Cardiovasc Surg. 2011 Feb;141(2): 463-8.

28. Lochbihler H, Hoelzl J, Dietz HG. Tissue compatibility and biodegradation of new absorbable stents for tracheal stabilization: an experimental study. J Pediatr Surg. 1997 May;32(5):717-20.

29. Korpela A, Aarnio P, Sariola H, Tormala P, Harjula A. Comparison of tissue reactions in the tracheal mucosa surrounding a bioabsorbable and silicone airway stents. Ann Thorac Surg. 1998 Nov;66(5): 1772-6.

30. Korpela A, Aarnio P, Sariola H, Tormala P, Harjula A. Bioabsorbable self-reinforced poly-L-lactide, metallic, and silicone stents in the man- agement of experimental tracheal stenosis. Chest. 1999 Feb;115(2): 490-5.

31. Robey TC, Valimaa T, Murphy HS, Tormala P, Mooney DJ, Weatherly RA. Use of internal bioabsorbable PLGA "finger-type" stents in a rabbit tracheal reconstruction model. Arch Otolaryngol Head Neck Surg. 2000 Aug;126(8):985-91.

32. Wang CE, Zhang PH. In vitro degradation behaviours of PDO monofilament and its intravascular stents with braided structure. Autex Res J. 2016;16(2):80-9.

33. Sabino MA, Gonzalez S, Marquez L, Feijoo JL. Study of the hydrolytic degradation of polydioxanone PPDX. Polym Degrad Stab. 2000;69(2):209-16.

34. Gefen A, Weihs D. Cytoskeleton and plasma-membrane damage resulting from exposure to sustained deformations: a review of the mechanobiology of chronic wounds. Med Eng Phys. 2016 Sep;38(9): 828-33.

35. Hill GR. Host $\gamma \delta$ T cells: an innate bridge to the epithelial targets of GVHD? Blood. 2005 Jul;106(2):393-4. 